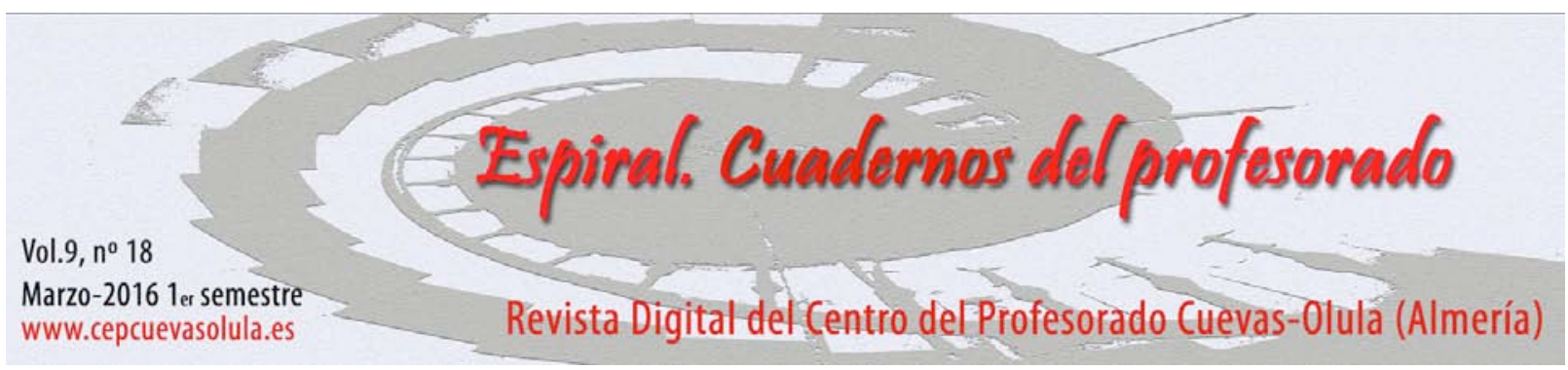

\title{
EL MODELO DE RESPONSABILIDAD PERSONAL Y SOCIAL A TRAVÉS DEL DEPORTE COMO PROPUESTA METODOLÓGICA PARA LA EDUCACIÓN EN VALORES EN ADOLESCENTES
}

Personal and Social Responsibility Model through Sport as methodological proposal for adolescents education in values

\section{Bernardino J. Sánchez-Alcaraz Martínez ${ }^{(1)}$, Alberto Gómez-Mármol ${ }^{(2)}$, Alfonso Valero Valenzuela ${ }^{(1)}$, Ernesto de la Cruz Sánchez ${ }^{(1)}$ y Arturo Díaz Suárez ${ }^{(1)}$}

(1) Universidad de Murcia, Facultad de Ciencias del Deporte, Murcia, España

(2) Universidad de Murcia, Facultad de Ciencias de la Educación, Murcia, España

RESUMEN: En este estudio se presenta el Modelo de Responsabilidad Personal y Social, desarrollado por Donald Hellison, como herramienta para la educación en valores y la mejora de la convivencia escolar a través de las clases de educación física y el deporte escolar. Se describe la metodología y las características del mismo, distinguiendo los cinco niveles de los que se compone (respeto por los demás, participación y esfuerzo, autonomía personal, ayuda a los demás y liderazgo y transferencia fuera del contexto deportivo), la estructura de las sesiones de intervención (toma de conciencia, la responsabilidad en acción, encuentro de grupo así como evaluación y autoevaluación) y pautas que debe seguir el educador en sus intervenciones. Del mismo modo, se ofrece una revisión actualizada de los principales trabajos de aplicación de este modelo, analizando sus características y los principales resultados obtenidos por los investigadores que han aplicado este Modelo tanto en España como en el extranjero.

Palabras clave: Educación Física, escolares, modelos de enseñanza, actividad física.

ABSTRACT: This paper presents the Personal and Social Responsibility Model, developed by Donald Hellison, as an instrument to improve the education in values and school life through physical education classes and school sports. The methodology, characteristics, distinguishing its five levels that compose it (respect for the others, participation and effort, personal autonomy, help the others and leadership and transferring outside the sport context), structure of intervention sessions (raise of awareness, responsibility in action, group meeting as well as assessment and self-assessment) and guidelines to be followed by the teacher in their interventions are described. In the same way, it provides a full review of the main works that are applied this model, and its characteristics and the main results obtained by the researchers who have applied this Responsibility Model in Spain and abroad.

Key words: Physical education, scholars, teaching models, physical activity.

Sánchez-Alcaraz, B. J., Gómez-Mármol, A., Valero Valenzuela, A., De la Cruz Sánchez, E., y Díaz Suárez, A. (2016). El Modelo de Responsabilidad Personal y Social a través del deporte como propuesta metodológica para la educación en valores en adolescentes. Espiral. Cuadernos del Profesorado, 9(18), 16-26. Disponible en: http://www.cepcuevasolula.es/espiral

Fecha de recepción: 31/07/2015

Fecha de aceptación: 11/01/2016
Enviar correspondencia a: alberto.gomez1@um.es 


\section{Introducción}

Los problemas de convivencia entre la población joven suponen un tema de debate actual en el seno de diversos países de la Unión Europea (Cangas, Gázquez, Pérez-Fuentes, Padilla, y Miras, 2007; López, 2004), debido al aumento, en los últimos años, del absentismo escolar acompañado de un clima de relación social en el que se localizan conductas relacionadas con intimidaciones, maltrato, xenofobia, agresiones o consumo de drogas en los centros escolares (Gázquez, Pérez-Fuentes, Carrión, y Santiuste, 2010; Ruiz et al., 2006).

Entre muchas de las intervenciones con el objeto de abordar esta problemática, las que se llevan a cabo en el contexto de la educación física y la enseñanza deportiva extraescolar han sido recurrentemente objeto de análisis (Ausín, Villaverde, y Lezcano Barbero, 2014), debido fundamentalmente a diferentes factores: en primer lugar, en estos entornos educativos es más sencillo el acceso a los adolescentes; en segundo lugar, la variedad y popularidad de estas actividades las hacen muy atractivas para gran parte de las personas en edad escolar; y por último, pero no menos importante, las características de estas actividades permiten que los jóvenes puedan establecer relaciones interpersonales muy abiertas con el educador y entre sus iguales (Escartí, Pascual, y Gutiérrez, 2005).

Sin embargo, el deporte y la educación física por sí mismos no disminuyen las conductas citadas anteriormente de forma automática (Carranza, y Mora, 2003), por lo que los cambios en los valores y las actitudes ocurren con más facilidad si son planteados de un modo sistemático y si tienen una orientación educativa en el propio contexto deportivo, ya que a pesar de que estos se desarrollan en ambientes atractivos e interactivos, emocionantes y divertidos para los jóvenes, sin la orientación pedagógica adecuada también pueden suponer en ocasiones entornos poco educativos (Cecchini, Montero, y Peña, 2003; Jaqueira, Lavega, Lagardera, Araujo, y Rodrigues, 2014).

De este modo, un programa físico-deportivo debería estar bien estructurado para que pueda constituir un instrumento efectivo de promoción de la responsabilidad personal y social, la deportividad y la disminución de la violencia en los jóvenes y que esta forma favorezca la convivencia aprovechando la alta interactividad y el carácter emocional de la actividad deportiva que permite enseñar valores y promover el desarrollo social y emocional (Martinek, Schilling, y Johnson, 2001).

\section{EI Modelo de Responsabilidad Personal y Social}

El Modelo de Responsabilidad Personal y Social (MRPS) fue creado y desarrollado por Donald Hellison, educador de la Universidad de Illinois (Chicago), hace más de treinta años. Aunque en sus inicios este fue concebido para trabajar los valores a través de la actividad física y el deporte con jóvenes socialmente desfavorecidos, en los últimos años este Modelo ha sido utilizado para el desarrollo de aspectos psicosociales tales como: respeto, autocontrol autoestima, empatía, esfuerzo, autonomía, cooperación, ayuda a los demás y liderazgo en otros contextos deportivos como las actividades extraescolares o entrenamientos y competiciones (Pardo, y García-Arjona, 2011). El Modelo de Responsabilidad ha sido desarrollado por su autor en numerosas publicaciones, destacando principalmente cuatro (Hellison, 1978, 1985, 1995, 2003).

\section{Los niveles de Responsabilidad}

Hellison (2003) propone cinco niveles de responsabilidad que se presentan a los estudiantes de modo progresivo y acumulativo y que definen comportamientos, actitudes y valores de responsabilidad personal y social para ser desarrollados a través de la actividad física y el deporte a partir de metas concretas y sencillas (Escartí et al., 2005). Junto a cada nivel existen una serie de estrategias y métodos específicos a desarrollar por los estudiantes y el profesorado y así poder alcanzar los objetivos que se plantean dentro de cada uno de los niveles del Modelo de Responsabilidad Personal y Social. Siguiendo a Hellison (2003), el propósito de todas las estrategias es mantener a los jóvenes interactuando con los niveles, no sólo para mejorar sus disciplina y motivación en el momento presente, sino lo que es más importante, que las actitudes y 
comportamientos desarrollados por ellos mismos se transfieran a otras áreas del gimnasio, siendo éste el objetivo último y también el más importante.

Nivel I: Respeto por los derechos y sentimientos de los demás.

En este nivel, el educador debe crear en el aula o en el gimnasio una atmósfera física y psicológicamente segura donde los participantes no se encuentren intimidados o amenazados por nadie, donde cada cual pueda manifestarse libremente sin temor a ser menospreciado o burlado (Escartí et al., 2005). Los siguientes dos aspectos relacionados con el respeto son especialmente aplicables dentro del ámbito físico-deportivo (Pardo, 2008):

- Respetar a los demás, sus opiniones, sus formas de ser y de actuar. Cualquier tipo de violencia física o verbal debe ser evitada, tratando de construir un clima adecuado de convivencia entre los estudiantes y con el docente. También hay que tener en cuenta el respeto hacia la figura de autoridad como puede ser el educador, el entrenador u otro compañero. Por otro lado, si un estudiante no realiza algún ejercicio correctamente, no debería ser recriminado por sus compañeros (o el educador) sino animado a intentarlo de nuevo.

- Respetar las reglas básicas de convivencia. Deben establecerse unas reglas comunes para ser respetadas por todos los participantes, facilitando de esta forma las relaciones personales, el clima de trabajo y la confianza. Estas reglas incluirían aspectos como: el respeto por el material y las instalaciones, asistencia regular a las clases/entrenamientos o la utilización de ropa deportiva adecuada, etc.

Estos dos aspectos anteriores están fuertemente ligados al autocontrol, ya que los educandos del nivel I no deben participar en las actividades propuestas si no son capaces de controlar su comportamiento lo suficiente como para no interferir en el derecho que tienen los otros educandos para aprender o el educador para enseñar (Hellison, 1995).

\section{Nivel II: Participación y esfuerzo.}

Una vez que el discente es capaz de mantener cierto autocontrol sobre su conducta pasa a formar parte de este segundo nivel, que supone la participación en las actividades de clase bajo la dirección del educador y que demanda esfuerzo y persistencia para no dejarse llevar por sentimientos de autoderrota y pasividad así como la presión negativa de sus compañeros. El objetivo de este nivel es ofrecer experiencias positivas a los educandos que participan en el Modelo para potenciar su implicación en el mismo y contribuir a que adquieran una visión positiva de la actividad física y del deporte que la conviertan en nuevo hábito positivo en sus vidas (Jiménez, 2000). Se trata de promover una participación sin discriminación, donde todos los educandos partan con igualdad de oportunidades, sin que la participación se supedite a características de sexo, niveles de habilidad u otros criterios discriminatorios como raza o cultura (Pardo, y García-Arjona, 2011).

\section{Nivel III. Autonomía personal:}

Este nivel trata de fomentar la autonomía del alumnado, es decir, regular su conducta por normas que surgen del propio individuo, enseñándoles a ser independientes y asumir sus responsabilidades (Escartí et al., 2005). Siguiendo a Pardo (2008), la toma de decisiones y la planificación son los dos componentes básicos en este nivel. Se trata de crear actitudes de responsabilidad ante las propias acciones y de fomentar estas capacidades que están estrechamente ligadas con la vida adulta. Por esta razón, es importante que en cada sesión del programa se propongan diferentes opciones en donde los educandos deban elegir y reflexionar después de ello. En cuanto a la planificación, Hellison (2003) destaca que los estudiantes en este nivel deberían ser capaces de elaborar y llevar a cabo un programa personal de actividad física. A través de la realización de este programa los discentes muestran que pueden ser responsables y autónomos a la hora de desarrollar sus propias actividades.

\section{Nivel IV: Ayuda a los demás y liderazgo.}

Este nivel se centra en dos importantes aspectos de la Responsabilidad Social: la empatía y el liderazgo. Para el desarrollo de la empatía los estudiantes deben ser conscientes de que los demás pueden tener un punto de vista diferente del suyo y tienen que aprender a respetarlo, poniéndose en el 
lugar del otro cuando sea necesario (Pardo, 2008). Al trabajar el liderazgo, el docente asignará a varios estudiantes el estar a cargo de un grupo para que trabajen de forma cohesionada y sea dicho líder el responsable de tomar las decisiones finales con respecto al grupo. En el nivel 4 se ponen como objetivos: conseguir ayudar a otros sin arrogancia y sin dogmatismo, ayudar sólo si otros precisan o quieren ayuda, tratando de evitar los comportamientos egocéntricos en los educandos y fomentar sus comportamientos solidarios.

\section{Nivel V: Fuera del contexto deportivo.}

Este último nivel enseña a los estudiantes a que apliquen a otros contextos de su vida (en otras clases, en el patio del recreo, en su casa, etc.) lo que han aprendido en los cuatro niveles del programa (Escartí et al., 2005). Los estudiantes que se encuentran en este Nivel muestran: respeto, esfuerzo, autonomía y liderazgo no sólo en el gimnasio, sino también fuera de él. Todo lo que ha ido aprendiendo el alumnado a lo largo del programa forma ya parte de sus vidas indistintamente de dónde se encuentren siendo, en definitiva, individuos responsables (Pardo, 2008).

\section{Estructura de la sesión educativa}

Durante el desarrollo del MRPS, las sesiones siguen una misma estructura con el objetivo principal de que los estudiantes puedan acostumbrarse a cumplir unas normas y que sepan exactamente qué se espera de ellos. De este modo, siguiendo a Hellison (2003), la sesión se divide en 4 partes principales:

Toma de conciencia: Durante los primeros 5 minutos el docente expone los comportamientos que deben aprender y practicar ese día, en concordancia con el Nivel de Responsabilidad que están trabajando. Una vez que los estudiantes terminan de entrar en el aula y se sientan en círculo, el docente expondrá de forma muy breve el objetivo educativo del día de forma que el alumnado sepa los comportamientos requeridos y los que no se van a consentir. A continuación, se explicará la actividad física a realizar y las normas para llevarlas a cabo.

La responsabilidad en acción: Durante esta fase (que ocupa la mayor parte del tiempo), es el momento de desarrollar las diferentes actividades físicas propuestas de acuerdo a los objetivos planteados y cuyo objetivo es que los estudiantes aprendan a comportarse con responsabilidad realizando los ejercicios planteados por el profesorado. Se deben planificar las actividades como vehículo para enseñar la Responsabilidad Personal y Social por lo que cualquier actividad puede ser útil, siempre que sea motivadora y tenga un significado de aprendizaje para el alumnado.

Encuentro de grupo: Al finalizar la actividad física, los estudiantes se sientan en círculo alrededor del docente y dedican un tiempo a compartir ideas, opiniones y pensamientos que han surgido durante la clase. Durante encuentro de grupo (que tiene una duración aproximada de 10 minutos), el docente lanzará una pregunta abierta orientada a preguntar a los educandos sobre cómo ha funcionado la actividad del día, qué han aprendido, si se han cumplido los objetivos, etc. La misión del profesorado en esta fase es conducir la dinámica del grupo haciendo preguntas y dejando tiempo suficiente para que los discentes contesten, elaborando el docente finalmente una pequeña conclusión sobre cómo ha ido la sesión y el modo de solucionar los problemas.

Evaluación y autoevaluación: Para concluir la sesión, el alumnado, sentado en círculo, durante dos o tres minutos, valora su comportamiento en clase en relación con el nivel del programa trabajado ese día. También evalúa el comportamiento de sus compañeros y del profesorado. Esta evaluación se realiza con un gesto de mano del siguiente modo: el dedo pulgar hacia arriba indica una evaluación positiva, el dedo pulgar hacia el lado indica una evaluación media y el dedo pulgar hacia abajo una evaluación negativa.

Es importante tener en cuenta lo imprescindible de respetar el formato de la sesión ya que el alumnado progresará más rápidamente cuando se les introducen rutinas bien estructuradas que ellos conocen y en las que se sienten más cómodos. 


\section{Investigaciones precedentes que han desarrollado programas de actividad física y deportiva, basados en el Modelo de Desarrollo de la Responsabilidad Personal y Social a través del deporte}

Siguiendo a Sánchez-Alcaraz, Díaz y Valero (2014), en estas tres últimas décadas han surgido diferentes programas de intervención basados en el Modelo de Responsabilidad Personal y Social de Hellison que, utilizando el deporte o la actividad física, han tenido como objetivo mejorar la convivencia y el desarrollo personal y social de niños y adolescentes. Estos programas se han llevado a cabo en diferentes modalidades, realizándose durante las clases de educación física, como actividades extraescolares o en campamentos ofertados por la escuela o los servicios sociales. Los objetivos de los diferentes programas han sido diversos aunque la mayoría de ellos se han centrado en incrementar en los sujetos el razonamiento moral, mejorar las atribuciones para el éxito y el fracaso, aumentar el autoconcepto y la autopercepción de eficacia y mejorar la concepción del mundo y de los demás. A continuación se presentan los más destacados:

\section{Investigaciones realizadas en el extranjero}

Estados Unidos es el país donde se han realizado la mayor parte de investigaciones relacionadas con el Modelo de Responsabilidad. Educadores de la University of Illinois (Chicago), California State University (Los Ángeles), University of Northen Colorado (Greeley), University of North Carolina (Geensboro) y University of Denver formaron una asociación llamada Urban Youth Leader Partnership, centrada en la promoción de programas físico-deportivos basados en el Modelo de Responsabilidad, dirigidos específicamente para los jóvenes de las áreas más deprimidas social y económicamente de las ciudades, buscando unir lazos entre la Universidad y las necesidades sociales del entorno en el que éstas se encuentran (Pardo, 2008). En este sentido, en el año 2000 han publicado de manera conjunta un libro titulado Youth development and physical activity: Linking universities and communities (Hellison et al., 2000), que es un referente indispensable para todos aquellos que quieran profundizar más acerca de cómo trabajar en valores con jóvenes socialmente desfavorecidos a través de la actividad física y el deporte (Pardo, 2008).

La primera investigación publicada sobre el Modelo de Responsabilidad corresponde a Hellison (1978) en su libro: Beyond balls and bats: alienated (and other) youth in the gym, en el que expone los objetivos y características de su Modelo de Responsabilidad para ser aplicado en jóvenes con problemas de disciplina y delincuencia. Posteriormente, los autores DeBusk y Hellison (1989) publicaron una investigación realizada aplicando esta metodología sobre 10 estudiantes en riesgo de abandono escolar que mejoraron el autocontrol y desarrollaron su plano cognitivo.

En la década de los 90 comienza la explosión de este tipo de programas. Georgiadis (1990) aplicó su programa con actividades relacionadas con el baloncesto en jóvenes que se encontraban en pisos de acogida, mejorando su autocontrol, la autonomía personal y la habilidad para trabajar en equipo. Posteriormente, junto a Hellison, a través del baloncesto mejoraron los valores de un grupo de jóvenes con problemas relacionados con el analfabetismo y las drogas (Hellison, y Georgiadis, 1992). Al mismo tiempo, Lifka (1990) publica una investigación aplicando el Modelo en jóvenes hispanoamericanos en riesgo de exclusión social, obteniendo mejoras en el plano cognitivo, la participación, el esfuerzo y la autonomía, además de una mejora en las relaciones interpersonales de los participantes.

Más tarde, Hellison (1995) trabaja con jóvenes en riesgo mejorando su responsabilidad personal y social a través de la aplicación del Modelo de Responsabilidad con actividades de baloncesto. En 2003, Hellison y Wright publican un trabajo que recoge 9 años de investigación y aplicación del MRPS en 78 jóvenes en riesgo que comenzaban el programa con 10-12 años. Una característica a destacar fue que los jóvenes de mayor edad adquirían el rol de líderes y responsables de grupos de menor edad, lo que les permitía trabajar las conductas de liderazgo. Los resultados de este estudio mostraron un aumento de las conductas de responsabilidad personal y de las habilidades sociales, así como la transferencia de estos comportamientos a otras esferas de su vida cotidiana (Hellison, y Wright, 2003). 
Es importante destacar los estudios de Cutforth en la Universidad de Denver, aplicados con jóvenes en riesgo, en los que a través de actividades como el baloncesto, mejoró la autoconfianza, el respeto a los demás, las habilidades interpersonales, las técnicas de comunicación y habilidades para resolver conflictos, el entusiasmo por el aprendizaje así como el concepto de transferencia, disminuyendo el número de expulsiones en otras asignaturas (Cutforth, 1997, 2000; Cutforth, y Parker, 1996; Cutforth, y Pucket, 1999).

Finalmente, es necesario referenciar los estudios de Tom Martinek a través del Proyecto Esfuerzo (Martinek, 1997; Martinek et al., 2001; Martinek, y Hellison, 1997; Martinek, McLaughlin, y Schilling, 1999; Martinek, y Ruiz, 2005; Martinek, y Schilling, 2003; Martinek, Schilling, y Hellison, 2006), aplicando el MRPS a niños con problemas de conducta y baja motivación hacia el entorno educativo a través de actividades deportivas variadas como fútbol, baloncesto, tenis, lacrosse, etc. Los resultados, tanto cualitativos como cuantitativos, reflejaron una mejora en el comportamiento de los educandos traducido en un menor número de conductas negativas, expulsiones $\mathrm{y} / \mathrm{o}$ abandonos (Martinek et al., 1999).

\section{Investigaciones realizadas en España}

En el año 2000, Escartí y su equipo de la Universidad de Valencia comienzan a desarrollar de manera ininterrumpida varias investigaciones basadas en el MRPS que recogen en el libro Responsabilidad Personal y Social a través de la educación física y el deporte (Escartí et al., 2005). En el año 2006, Escartí et al. registraron los efectos de la aplicación de este Modelo en los comportamientos de responsabilidad social (agredir, interrumpir, no colaborar e interrumpir) en 13 adolescentes miembros del Programa de Adaptación Curricular en Grupo de $4^{\circ}$ de ESO, apreciando una reducción de las conductas agresivas y de interrupción, mientras que las de no colaborar e interrumpir se mantuvieron estables.

Posteriormente, Escartí, Gutiérrez, Pascual y Llopis (2010) han aplicado el MRPS en las clases de educación física en un grupo de 42 educandos de 11 y 12 de años pertenecientes a sexto de Educación Primaria, cuyos resultados han mostrado un incremento significativo de la autoeficacia en los participantes. Ese mismo año, Escartí, Gutiérrez, Pascual y Marín (2010) implementaron el MRPS en un grupo de 30 adolescentes de 13 y 14 años de edad en riesgo de abandono escolar y cuyos resultados mostraron mejoras significativas en la autoeficacia para conseguir recursos sociales y en la autoeficacia para el aprendizaje regulado del alumnado, así como una percepción de mejora en la conducta responsable de los educandos y en la responsabilidad de sus iguales.

Del mismo modo, Pascual, Escartí, Llopis, y Gutiérrez (2011) han descrito los efectos observados por cinco maestros de educación física en sus educandos de Educación Primaria tras implementar el MRPS en sus clases durante dos cursos escolares, percibiendo todos los maestros participantes beneficios sobre la gran mayoría de sus discentes en los objetivos referentes al nivel 1 "respetar los derechos y sentimientos de los demás", a saber: resolver los conflictos a través del diálogo, evitar exclusiones de los compañeros y hablar respetando el turno de palabra. Además, observaron que los efectos del MRPS son mayores en aquellos discentes que no tienen unos modelos de comportamiento bien interiorizados, sean estos positivos o negativos (Pascual et al., 2011).

Asimismo, Gutiérrez, Escartí, y Pascual (2011) han analizado las relaciones de la responsabilidad personal y social con las variables de empatía, conducta prosocial, agresividad y autoeficacia en un grupo de 822 educandos de 8 a 15 años. Los resultados han mostrado que la conducta prosocial, empatía y percepción de eficacia predicen positivamente la responsabilidad personal y social de los escolares, mientras que la agresividad se asocia negativamente con la responsabilidad.

Por otro lado, el grupo de trabajo dirigido por Cecchini desde la Universidad de Oviedo lleva trabajando varios años en proyectos basados en el MRPS, y si bien sus trabajos no se centran, como la mayoría de las anteriores, en jóvenes desfavorecidos, han obtenido resultados significativos en las conductas relacionadas con el autocontrol y la deportividad o "fair play".

De este modo, en 2003 han examinado las repercusiones del MRPS en una muestra de 142 estudiantes en Educación Primaria durante una unidad didáctica (10 sesiones) de iniciación al fútbol 
sala, mostrando mejoras significativas en la retroalimentación personal, el retraso de la recompensa, el auto-control criterial, el auto-control del proceso, las opiniones relacionadas con la diversión y las conductas deportivas (Cecchini et al., 2003). Del mismo modo, observaron disminuciones en las variables relacionadas con el juego duro, las faltas de contacto y las conductas antideportivas. Posteriormente, Cecchini, Montero, Alonso, Izquierdo y Contreras (2007) han analizado la utilidad del MRPS en 186 escolares de Educación Secundaria Obligatoria durante 10 clases de educación física sobre fútbol sala, mejorando significativamente la responsabilidad personal y social, el autocontrol y la deportividad y disminuyendo las conductas agresivas como faltas de contacto. Posteriormente a este trabajo, los mismos autores (Cecchini et al., 2009) han examinado a través del Programa Delfos la repercusión del MRPS en los niveles de agresividad en un grupo de 160 estudiantes de Educación Primaria durante 24 sesiones de educación física, mostrando mejoras significativas en los comportamientos asertivos y una disminución de conductas agresivas tanto en el deporte como en otros contextos.

Igualmente, cabe destacar el trabajo realizado por Ruiz desde la Universidad de Castilla La Mancha, en colaboración con Tom Martinek, dando a conocer el Project Effort (Proyecto Esfuerzo) y que está fundamentado en el Modelo de Responsabilidad Personal y Social (Martinek, y Ruiz, 2005; Ruiz et al., 2006) o la investigación desarrollada por Pardo (2008) aplicando el Modelo de Responsabilidad durante 10 semanas en tres centros educativos situados en Getafe (España), L'Aquila (Italia) y Los Ángeles (Estados Unidos) sobre un total de 51 educandos con edades comprendidas entre los 15 y 19 años, procedentes de 11 países diferentes. Su estudio ha mostrado un cambio progresivo en el comportamiento de los educandos más problemáticos así como una mejora en las actitudes de respeto, participación y esfuerzo y autonomía personal.

Finalmente, es preciso mencionar los trabajos que se están desarrollando desde la Universidad de Murcia. En este sentido Sánchez-Alcaraz, Gómez-Mármol, Valero, De la Cruz y Esteban (2012), aplicaron el MRPS en 802 escolares mejorando la calidad de vida de los estudiantes de Educación Secundaria. Posteriormente Sánchez-Alcaraz, Gómez-Mármol, Valero y De la Cruz (2013) mejoraron la responsabilidad personal y social en un grupo de 186 estudiantes a través del MRPS aplicado en las clases de Educación Física.

\section{Conclusiones}

El MRPS ha mostrado sus beneficios durante los treinta años que lleva implantándose, tanto en jóvenes con situación de riesgo social como en escolares. Todas las investigaciones descritas anteriormente se adjuntan a continuación en la tabla 1, en la que se resaltan sus características más reseñables como los participantes, la duración de la implementación del Modelo, el contexto de aplicación y los resultados más importantes. Finalmente, como características comunes de las investigaciones desarrolladas, la mayoría han sido aplicadas en escolares de educación primaria, durante programas extraescolares, con actividades propias tanto de la educación física como del deporte escolar. La mayoría de los programas han tenido una duración de tres meses aproximadamente aunque algunos se han llegado a alargar hasta un curso académico y la metodología utilizada para valorar el impacto en los participantes ha sido predominantemente cualitativa. Los resultados de los diferentes estudios de intervención han descrito cómo el MRPS se erige como una metodología que permite la educación en valores de los escolares a través de las clases de educación física, adquiriendo conductas tan importantes como: respeto, autocontrol autoestima, empatía, esfuerzo, autonomía, cooperación, ayuda a los demás y liderazgo. 
Tabla 1.

Resumen de las investigaciones más importantes que aplican el Modelo de Responsabilidad Personal y Social.

\begin{tabular}{|c|c|c|c|c|}
\hline Autores & Muestra & Duración & Contexto & Resultados \\
\hline $\begin{array}{l}\text { DeBusk y } \\
\text { Hellison } \\
(1989)\end{array}$ & $\begin{array}{l}10 \text { estudiantes } \\
\text { de } 9 \text { y } 10 \text { años }\end{array}$ & 3 meses & $\begin{array}{l}\text { Actividades deportivas } \\
\text { enfatizadas en voleibol } \\
\text { para jóvenes con } \\
\text { problemas de conducta. }\end{array}$ & $\begin{array}{l}\text { El modelo causó cambios } \\
\text { afectivos, de comportamiento y de } \\
\text { conocimiento en los participantes. }\end{array}$ \\
\hline $\begin{array}{l}\text { Georgiadis } \\
(1990)\end{array}$ & $\begin{array}{l}\text { Jóvenes de } \\
\text { entre } 14 \text { y } 17 \\
\text { años }\end{array}$ & 3 meses & $\begin{array}{l}\text { Actividades de baloncesto } \\
\text { para jóvenes en pisos de } \\
\text { acogida. }\end{array}$ & $\begin{array}{l}\text { Mejoras en el autocontrol, la } \\
\text { autonomía personal y la habilidad } \\
\text { para trabajar en equipo. }\end{array}$ \\
\hline Lifka (1990) & $\begin{array}{l}\text { Jóvenes de } \\
\text { entre } 11 \text { y } 13 \\
\text { años }\end{array}$ & 4 meses & $\begin{array}{l}\text { Actividades físicas y } \\
\text { voleibol con minorías } \\
\text { hispanoamericanas en } \\
\text { riesgo de exclusión social. }\end{array}$ & $\begin{array}{l}\text { Mejoras en el plano cognitivo, la } \\
\text { participación, el esfuerzo, la } \\
\text { autonomía y las relaciones } \\
\text { interpersonales de los } \\
\text { participantes. }\end{array}$ \\
\hline $\begin{array}{l}\text { Hellison } \\
\text { (1993) }\end{array}$ & $\begin{array}{l}\text { Jóvenes de } \\
\text { entre } 11 \text { y } 13 \\
\text { años }\end{array}$ & 6 meses & $\begin{array}{l}\text { Actividades de baloncesto } \\
\text { con jóvenes en riesgo. }\end{array}$ & $\begin{array}{l}\text { Mejoras en la responsabilidad } \\
\text { personal y social.. }\end{array}$ \\
\hline $\begin{array}{l}\text { Cutforth } \\
\text { (1997) }\end{array}$ & $\begin{array}{l}\text { Varios grupos } \\
\text { de estudiantes } \\
\text { de entre } 11 \mathrm{y} \\
13 \text { años }\end{array}$ & 24 meses & $\begin{array}{l}\text { Actividades deportivas } \\
\text { con jóvenes } \\
\text { afroamericanos }\end{array}$ & $\begin{array}{l}\text { Mejoras en el comportamiento, las } \\
\text { técnicas de comunicación y las } \\
\text { relaciones interpersonales y } \\
\text { disminución de expulsiones y } \\
\text { abandonos.' }\end{array}$ \\
\hline $\begin{array}{c}\text { Cutforth y } \\
\text { Pucket (1999) }\end{array}$ & $\begin{array}{l}11 \text { escolares } \\
\text { entre } 10 \text { y } 13 \\
\text { años }\end{array}$ & 6 semanas & Actividades de baloncesto & $\begin{array}{l}\text { Mejora de la autoconfianza, el } \\
\text { respeto a los demás, las } \\
\text { habilidades para resolver } \\
\text { conflictos y habilidades } \\
\text { interpersonales y el entusiasmo } \\
\text { por el aprendizaje. }\end{array}$ \\
\hline $\begin{array}{l}\text { Cecchini, } \\
\text { Montero y } \\
\text { Peña }(2003)\end{array}$ & $\begin{array}{l}142 \text { escolares } \\
\text { de } 12 \text { y } 13 \\
\text { años }\end{array}$ & 2 meses & $\begin{array}{l}\text { Una unidad didáctica de } \\
\text { fútbol sala durante las } \\
\text { clases de educación física. }\end{array}$ & $\begin{array}{l}\text { Mejoras en la retroalimentación } \\
\text { personal, el retraso a la } \\
\text { recompensa, el autocontrol y la } \\
\text { deportividad. Descenso del juego } \\
\text { duro y faltas de contacto. }\end{array}$ \\
\hline $\begin{array}{c}\text { Hellison y } \\
\text { Wright (2003) }\end{array}$ & $\begin{array}{l}78 \text { jóvenes de } \\
10 \text { a } 19 \text { años }\end{array}$ & 9 años & $\begin{array}{l}\text { Actividades deportivas } \\
\text { con jóvenes en riesgo }\end{array}$ & $\begin{array}{l}\text { Mejoras en la responsabilidad } \\
\text { personal y social y conductas de } \\
\text { liderazgo, así como transferencia } \\
\text { de comportamientos a otros } \\
\text { contextos. }\end{array}$ \\
\hline $\begin{array}{l}\text { Escartí et al. } \\
\qquad(2006)\end{array}$ & $\begin{array}{c}13 \\
\text { adolescentes } \\
\text { de } 15-16 \text { años }\end{array}$ & 3 meses & $\begin{array}{l}\text { Clases de educación } \\
\text { física. }\end{array}$ & $\begin{array}{l}\text { Reducción de las conductas } \\
\text { agresivas y de interrupción. }\end{array}$ \\
\hline $\begin{array}{l}\text { Cecchini, } \\
\text { Montero, } \\
\text { Alonso, } \\
\text { Izquierdo y }\end{array}$ & $\begin{array}{l}186 \\
\text { estudiantes de } \\
3^{\circ} \text { ESO (13- } \\
14 \text { años) }\end{array}$ & 2 meses & $\begin{array}{l}\text { Una unidad didáctica de } \\
\text { fútbol sala durante las } \\
\text { clases de educación física. }\end{array}$ & $\begin{array}{l}\text { Mejoras en la responsabilidad } \\
\text { personal y social, el autocontrol y } \\
\text { la deportividad. Disminución del } \\
\text { juego duro y las faltas de contacto. }\end{array}$ \\
\hline
\end{tabular}

(2007)

Pardo (2008)

51 escolares entre 15 y 19
10 semanas en cada
Clases de educación física en tres centros escolares
Mejoras en las actitudes de respeto, participación y esfuerzo y 


\begin{tabular}{|c|c|c|c|c|}
\hline & años & centro & $\begin{array}{l}\text { con jóvenes en riesgo en } \\
\text { España, Italia y EEUU. }\end{array}$ & autonomía personal. \\
\hline $\begin{array}{l}\text { Cecchini et al. } \\
\text { (2009) }\end{array}$ & $\begin{array}{l}160 \\
\text { estudiantes de } \\
5^{\mathrm{a}} \text { y } 6^{\mathrm{a}} \text { de } \\
\text { Primaria (10- } \\
12 \text { años) }\end{array}$ & 3 meses & $\begin{array}{l}\text { Clases de educación } \\
\text { física. }\end{array}$ & $\begin{array}{l}\text { Mejoras significativas en los } \\
\text { comportamientos asertivos y } \\
\text { disminución de las conductas } \\
\text { agresivas tanto en el deporte como } \\
\text { en otros contextos. }\end{array}$ \\
\hline $\begin{array}{l}\text { Escartí, } \\
\text { Gutiérrez, } \\
\text { Pascual y } \\
\text { Llopis }(2010)\end{array}$ & $\begin{array}{l}42 \text { escolares } \\
\text { de } 11 \text { y } 12 \\
\text { años }\end{array}$ & $\begin{array}{c}1 \text { año } \\
\text { académico }\end{array}$ & $\begin{array}{l}\text { Clases de educación } \\
\text { física. }\end{array}$ & $\begin{array}{l}\text { Mejoras en los comportamientos } \\
\text { de responsabilidad y un } \\
\text { incremento significativo de la } \\
\text { autoeficacia. }\end{array}$ \\
\hline $\begin{array}{c}\text { Escartí, } \\
\text { Gutiérrez, } \\
\text { Pascual y } \\
\text { Marín (2010) }\end{array}$ & $\begin{array}{l}30 \text { escolares } \\
\text { de } 3^{\circ} \text { de ESO } \\
(13-14 \text { años })\end{array}$ & $\begin{array}{c}1 \text { año } \\
\text { académico }\end{array}$ & $\begin{array}{l}\text { Clases de educación física } \\
\text { con adolescentes en } \\
\text { riesgo de abandono } \\
\text { escolar. }\end{array}$ & $\begin{array}{l}\text { Mejoras en las conductas } \\
\text { responsables, la responsabilidad } \\
\text { con sus iguales y en la } \\
\text { autoeficacia del alumnado. }\end{array}$ \\
\hline $\begin{array}{l}\text { Pascual, } \\
\text { Escartí, Llopis } \\
\text { y Gutiérrez } \\
(2011)\end{array}$ & $\begin{array}{l}140 \text { escolares } \\
\text { de } 5^{\circ} \text { de } \\
\text { Primaria }(10- \\
11 \text { años })\end{array}$ & $\begin{array}{l}2 \text { cursos } \\
\text { académicos }\end{array}$ & Clases de educación física & $\begin{array}{l}\text { Percepción de los maestros de } \\
\text { beneficios en la mayoría de los } \\
\text { participantes, principalmente en la } \\
\text { variable de respeto a los demás. }\end{array}$ \\
\hline $\begin{array}{c}\text { Sánchez- } \\
\text { Alcaraz, } \\
\text { Gómez- } \\
\text { Marmol, } \\
\text { Valero, De la } \\
\text { Cruz y Díaz } \\
\text { (2012) }\end{array}$ & $\begin{array}{l}802 \\
\text { estudiantes de } \\
6^{\circ} \text { Primaria y } \\
3^{\circ} \text { ESO }\end{array}$ & 4 meses & Clases de educación física & $\begin{array}{l}\text { Mejora de la calidad de vida en } \\
\text { los discentes de Educación } \\
\text { Secundaria }\end{array}$ \\
\hline $\begin{array}{c}\text { Sánchez- } \\
\text { Alcaraz, } \\
\text { Gómez- } \\
\text { Marmol, } \\
\text { Valero y De la } \\
\text { Cruz (2013) }\end{array}$ & $\begin{array}{l}186 \\
\text { estudiantes de } \\
6^{\circ} \text { Primaria y } \\
3^{\circ} \text { ESO }\end{array}$ & $\begin{array}{c}24 \text { sesiones } \\
(3 \text { meses })\end{array}$ & Clases de educación física & $\begin{array}{l}\text { Mejora significativa de la } \\
\text { responsabilidad personal y social } \\
\text { en el alumnado de primaria y de } \\
\text { secundaria }\end{array}$ \\
\hline
\end{tabular}

Fuente: Elaboración propia

\section{Notas}

Investigación financiada por la Consejería de Educación, Formación y Empleo de la Comunidad Autónoma de la Región de Murcia. Proyecto de Innovación perteneciente al Programa III, resolución 593/2009 de fecha 20 de noviembre de 2009, del Rectorado de la Universidad de Murcia. Título: El deporte y la actividad física como medio para el desarrollo personal y social en jóvenes escolares.

\section{Referencias}

Ausín Villaverde, V. y Lezcano Barbero, F. (2014). Programas para la inclusión educativa de alumnado de origen extranjero en España: diseño y validación. Espiral: Cuadernos del profesorado, 7 (13), 31-43.

Cangas, A., Gázquez, J., Pérez-Fuentes, M. C., Padilla, D., y Miras, F. (2007). Evaluación de la violencia escolar y su afectación personal en una muestra de escolares europeos. Psicothema, 19 (1), 114-119.

Carranza, M. y Mora, J. M. (2003). Educación fisica y valores: educando en un mundo complejo. Barcelona: Graó.

Cecchini, J. A., González, C., Alonso, C., Barreal, J. M., Fernández, C., García, M., Llaneza, R., y Nuño, P. (2009). Repercusiones del Programa Delfos sobre los niveles de agresividad en el deporte y otros contextos de la vida diaria. Apunts, Educación Física y Deportes, 96, 34-41. 
Cecchini, J. A., Montero, J., Alonso, A., Izquierdo, M., y Contreras, O. (2007). Effects of Personal and Social Responsibility on fair play in sports and self-control in school-aged youths. European Journal of Sport Science, 7 (4), 203-211.

Cecchini, J. A., Montero, J., y Peña. J. V. (2003). Repercusiones del programa de intervención para desarrollar la responsabilidad personal y social de Hellison sobre los comportamientos de fair-play y el auto-control. Psicothema, 15, 631-637. Cu

Cutforth, N. (1997). What's worth doing: reflections on an after-school program in a Denver Elementary School. Quest, 49, 130-139.

Cutforth, N. (2000). Connecting school physical education to the community through service-learning. Journal of Physical Education, Recreation and Dance, 71 (2), 39-45.

Cutforth, N. y Parker, M. (1996). Promoting affective development in physical education: The value of journal writing. Journal of Physical Education, Recreation and Dance, 57 (7), 19-23.

Cutforth, N. y Pucket, K. M. (1999). An investigation into the organization, challenges, and impact of an urban apprentice teacher program. The Urban Review, 31 (2), 153-172.

Debusk, M. y Hellison, D. (1997). Implementing a Physical Educatoin Self-Responsibility Model for Delinquency-Prone Youth. Journal of Teaching in Physical Education, 8, 104-112.

Escartí, A., Gutiérrez, M., Pascual, C., y Llopis, R. (2010). Implementation of the Personal and Social Responsibity Model to improve self-efficacy during physical education classes for primary school children. International Journal of Psychology and Phychological Therapy, 10 (3), 387-402.

Escartí, A., Gutiérrez, M., Pascual, C., y Marín, D. (2010). Application of Hellison's Teaching Personal and Social Responsibility Model in Physical Education to improve self-efficacy for adolescents at risk of dropping-out of school. The Spanish Journal of Psychology, 13 (2), 667-676.

Escartí, A., Gutiérrez, M., Pascual, C., Marín, D., Martínez, C., y Chacón, Y. (2006). Enseñando responsabilidad personal y social a un grupo de adolescentes de riesgo: un estudio "observacional". Revista de Educación, 341, 373-396.

Escartí, A., Pascual, C., y Gutiérrez, M. (2005). Responsabilidad Personal y Social a través de la educación física y el deporte. Barcelona: Graó.

Gázquez, J. J., Pérez-Fuentes, M. C., Carrión, J. J., y Santiuste, V. (2010). Estudio y análisis de conductas violentas en Educación Secundaria Obligatoria en España. Universitas Psychologica, 9 (2), 371-380.

Georgiadis, N. (1990). Does basketball have to be all W's and L's? An alternative program at a residential boys' home. Journal of Physical Education, Recreation and Dance, 61 (6). 42-43.

Gutiérrez, M. Escartí, A., y Pascual, C. (2011). Relaciones entre empatía, conducta prosocial, agresividad, autoeficacia y responsabilidad personal y social. Psicothema, 23 (1), 13-19.

Hellison, D. (1978). Beyond balls and bats: allienated (and other) youth in the gym. Washington DC: AAHPER publications.

Hellison, D. (1985). Goal and strategies for teaching physical education. Champaign, IL.: Human Kinetics.

Hellison, D. (1995). Teaching responsibility through physical activity. Champaign, IL: Human Kinetics.

Hellison, D. (2003). Teaching responsibility through physical activity (2 ${ }^{\mathrm{a}}$ ed.). Champaign, IL: Human Kinetics.

Hellison, D., Cutforth, N., Kallusky, J., Martinek, T., Parker, M., y Stiehl, J. (2000). Youth development and physical activity: Linking universities and communities. Champaign, IL: Human Kinetics.

Hellison, D. y Georgiadis, N. (1992). Teaching values through basketball. Strategies: A Journal for Physical and Sport Educators, 5 (4), 5-8.

Hellison, D. y Wright, P. M. (2003). Retention in an urban extended day program: A process-based assessment. Journal of Teaching in Physical Education, 22 (4), 369-381.

Jaqueira, A. R., Lavega, P., Lagardera, F., Aráujo, P., y Rodrigues, M. (2014). Educando para la paz jugando: género y emociones en la práctica de juegos cooperativos competitivos. Educatio Siglo XXI, 32 (2), 15 32.

Jiménez, P. (2000). Modelo de intervención para educar en valores a jóvenes en riesgo a través de la actividad física y el deporte. Tesis Doctoral. Universidad Politécnica de Madrid, Madrid, España.

Lifka, B. (1990). Hiding beneath the Stairwell-A dropout prevention program for hispanic youth. Journal of Physical Education, Recreation and Dance, 61 (6), 40-41.

López, H. (2004). Padres y alumnos ante el valor de responsabilidad. Educatio Siglo XXI, 22, 187-205. 
Martinek, T. (1997). Serving underserved youth through physical activity. Quest, 49, 3-7.

Martinek, T. \& Hellison, D. (1997). Fostering resiliency in underserved youth through physical activity. Quest, 49, 34-49..

Martinek, T., McLaughlin, D., y Schilling, T. (1999). Project Effort: Teaching responsibility beyond the gym. Journal of Phyisical Education, Recreation and Dance, 70 (6), 59-65.

Martinek, T. y Ruiz, M. (2005). Promoting positive youth development through a values-based sport program. Revista Internacional de Ciencias del Deporte, 1 (1), 1-13.

Martinek, T. y Schilling, T. (2003). Developing compassionate leadership in underserved youths. Jorunal of Physical Education, Recreation and Dance, 74 (5), 33-39.

Martinek, T., Schilling, T., y Hellison, D. (2006). The development of compassionate and caring leadership among adolescents. Physical Education and Sport Pedagogy, 11 (2), 141-157.

Martinek, T., Schilling, T., y Johnson, D. (2001). Transferring personal and social responsibility of underserved youth to the classroom. The Urban Review, 33 (1), 29-45.

Pardo, R. (2008). La transmisión de valores a jóvenes socialmente desfavorecidos a través de la actividad fisica y el deporte. Estudio Múltiple de Casos: Getáfe, L'Aquila y Los Ángeles. Tesis Doctoral Europea, Universidad Politécnica de Madrid.

Pardo, R. y García-Arjona, N. (2011). El Modelo de Responsabilidad: desarrollo de aspectos psicosociales en jóvenes socialmente desfavorecidos a través de la actividad física y el deporte. Revista de Psicología y Educación, 6, 211-222.

Pardo, C., Escartí, A., Llopis, R., y Gutiérrez, M. (2011). La percepción del profesorado de educación física sobre los efectos del Programa de Responsabilidad Personal y Social en los estudiantes. Ágora para la educación fisica y el deporte, 13 (3), 341-361.

Ruiz, L. M., Rodríguez, P., Martinek, T., Schilling, T., Durán, L. J., y Jiménez, P. (2006). El proyecto esfuerzo: un modelo para el desarrollo de la responsabilidad personal y social a través del deporte. Revista de Educación, 341, 933-958.

Sánchez-Alcaraz, B. J., Díaz, A., y Valero, A. (2014). Mejora de la convivencia escolar a través de la Educación Física. El Modelo de Responsabilidad Personal y Social. Saarbücken, Deutschland: Editorial Académica Española.

Sánchez-Alcaraz, B. J., Gómez-Mármol, A., Valero, A., y De La Cruz, E. (2013). Aplicación de un programa para la mejora de la responsabilidad personal y social en las clases de Educación Física. Motricidad. European Journal of Human Movement, 30, 121-129.

Sánchez-Alcaraz, B. J., Gómez-Mármol, A., Valero, A., De La Cruz, E., y Esteban, R. (2012). Influencia del Modelo de Responsabilidad Personal y Social en la Calidad de Vida de los Escolares. Cuadernos de Psicología del Deporte, 12 (2), 13-18. 